\title{
Estimation of the spatial resolution of a 2D strain estimator using synthetic cardiac images
}

\author{
Bidisha Chakraborty*, Sophie Giffard-Roisin ${ }^{\dagger}$, Martino Alessandrini*, Brecht Heyde*, Maxime Sermesant ${ }^{\ddagger}$, Jan D’hooge* \\ ${ }^{*}$ Cardiovascular Imaging and Dynamics, KU Leuven, Leuven, Belgium \\ ${ }^{\dagger}$ CNRS, Université Paris-Sud, Laboratoire d'Accélérateur Linéaire, Paris, France \\ ‡Inria, Université Cote d’Azur, Nice, France \\ Email: bidisha.chakraborty@kuleuven.be
}

\begin{abstract}
Although myocardial deformation imaging is often used in clinical practice, to date, the spatial resolution of any speckle tracking algorithm has not been tested. The minimum size of the resolvable dysfunctional areas with these techniques remains unknown while this is relevant for reliable clinical diagnosis. The current study therefore aimed at determining the spatial resolution of our previously validated non-rigid image registration (NRIR) algorithm in 2D ultra-realistic in-silico Bmode images with eight varying sizes of dysfunctional regions. A correlation coefficient obtained between the NRIR-end-systolic (ES) strains and the true ones was 0.97 , indicating a good tracking performance. Mean ES strain errors and limits of agreement were $0.19 \%$ and $-3.37 \cdots 3.76 \%$ respectively $(p<0.01)$. Based on the pixel-wise ES strain differences between the normal heart model and the model with dysfunctional regions, the sensitivity, specificity and accuracy to detect dysfunction were computed, where a region was considered resolved if more than $>60 \%$ of the pixels were correctly classified. Furthermore, a correlation coefficient of 0.92 as determined by the correlation between the NRIR-estimated sizes of the dysfunctional regions against the ground truth sizes is suggestive of good tracking resolution. In this way our study revealed that the discriminative power of our algorithm was adequate to resolve dysfunctional areas down to $1.9 \mathrm{~cm}$.
\end{abstract}

\section{INTRODUCTION}

Myocardial deformation imaging is often used for noninvasive quantification of left ventricular regional function in clinical cardiology. Although regional strain analysis is widely used in clinics, to date, the spatial resolution of speckle tracking algorithms has not been tested. As such, the minimum size of resolvable dysfunctional area with these techniques remains unknown while this is relevant for reliable clinical diagnosis.

Commercial speckle tracking solutions are almost exclusively based on block matching. As an alternative methodology, our group has previously validated non-rigid image registration (NRIR) in 2D in-silico cardiac images [1] and NRIR was reported to be the potentially more robust tracking solution. However, the spatial resolution of NRIR has never been determined. The aim of the current study was therefore to investigate the spatial resolution of NRIR based strain estimates in ultra-realistic 2D in-silico cardiac images, simulating varying sizes of dysfunctional regions.

\section{MATERIALS AND METHODS}

Data

Ultra-realistic 2D synthetic B-mode apical images (A3C and A4C) were generated by combining a complex electromechanical (E/M) model of the heart with a novel ultrasound (US) simulation approach [2]. Specifically, the Bestel-ClementSorine E/M model [3] was used to generate the synthetic motion field across the whole cardiac cycle. Next to a model mimicking a normal heart, 8 data sets with dysfunctional areas ranging from $1.6 \mathrm{~cm}$ to $5.2 \mathrm{~cm}$ in diameter were generated by locally modifying biophysical parameters such as the active contractile force, stiffness and conduction. The dysfunctional regions located on the lateral wall lying in between the $\mathrm{A} 3 \mathrm{C}$ and $\mathrm{A} 4 \mathrm{C}$ images were simulated with realistic extent and shapes as generated in [4]. Furthermore, the ventricle geometry and realistic speckle textures were obtained from the templates of real clinical US recordings [2].

\section{Non-rigid image registration}

Non-rigid image registration computes the inter-frame displacement field $\mathbf{T}_{\xi}=\left(\mathrm{T}_{\mathrm{x}}, \mathrm{T}_{\mathrm{y}}\right)$ at every pixel $\mathrm{r}=[\mathrm{x}, \mathrm{y}]$ and it was modeled as a tensor product of $2 \mathrm{D}$ cubic B-splines $\beta_{\xi}^{3}$ $(\xi \in(\mathrm{x}, \mathrm{y}))[5]:$

$$
\mathbf{T}_{\xi}(\mathrm{r})=\sum_{\mathrm{i} \in \mathrm{N}_{\mathrm{i}}} \sum_{\mathrm{j} \in \mathrm{N}_{\mathrm{j}}} \mu_{\mathrm{ij}} \beta_{\mathrm{x}}^{3}\left(\frac{\mathrm{x}-\kappa_{\mathrm{x}}^{\mathrm{ij}}}{\sigma_{\mathrm{x}}}\right) \beta_{\mathrm{y}}^{3}\left(\frac{\mathrm{y}-\kappa_{\mathrm{y}}^{\mathrm{ij}}}{\sigma_{\mathrm{y}}}\right)
$$

where within the compact support of the cubic B-splines $\left(\beta_{\xi}^{3}\right)$ lies a set of control grid points $\mathrm{N}_{\xi}$, with an inter-grid spacing of $\sigma_{\xi}$.

$\mathbf{T}_{\xi}(\mathrm{r})$ was parametrized by B-spline coefficients $\mu_{\mathrm{ij}}$, located at every control point node. During an iterative optimization process, these coefficients were updated to optimum values by minimizing a cost function.

In a multi-resolution framework [5], [6], firstly the transformation complexity was gradually increased by progressively decreasing the inter-grid spacings, $\sigma_{\xi}$ between control points in subsequent levels. Secondly, the data complexity was gradually increased by convolving the images with a Gaussian scale space, where the size of the smoothing kernel was reduced at each scale. 


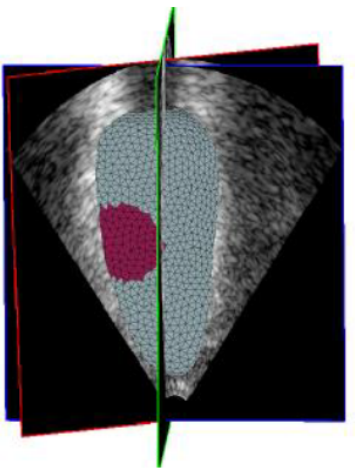

a)

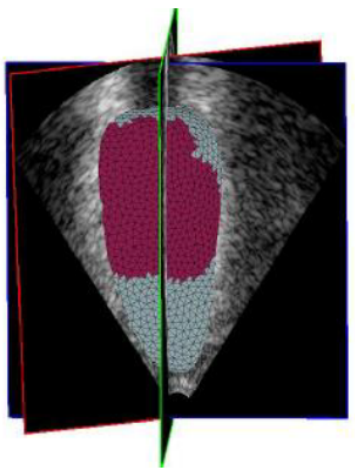

b)
Figure 1. Visual representation of 3D meshes where (a) the smallest and (b) the largest dysfunctional regions are represented by the red parts. The A3C intersecting slice is shown by the green imaging plane.

This large number of coefficients $\mu_{\mathrm{ij}}$, were optimized by a limited memory Broyden-Fletcher-Goldfarb-Shanno (LBFGSB) optimization routine with simple bounds, generally suitable for optimization of a large set of parameters due to its low memory requirement [7].

The cost function used for optimization can be expressed as:

$$
C=\mathrm{S}+\omega \mathrm{R}
$$

where $\mathrm{S}$ is a similarity measure and $\mathrm{R}$ is a regularization term, weighted by a factor $\omega$ to adjust the strength of R. Here, the sum of squared differences (SSD) has been chosen as the similarity measure $\mathrm{S}$, primarily due to its good performance in monomodal registration and computational simplicity. Additionally, physically impossible solutions of the displacement field $\left(\mathbf{T}_{\xi}\right)$ were penalized by computation of a bending energy (BE) penalty term which was derived as a squared second order derivative of $\mathbf{T}_{\xi}$ and represented here by $\mathrm{R}$ [5].

Conventionally, the $\mathrm{BE}$ penalty term is computed by the second order derivatives of the underlying transformation field at every pixel location, $\mathrm{r}$ in the image consisting of $\mathrm{P}$ pixels, therefore making the computational complexity proportional to the total number of pixels $(\mathrm{P})$ present in the image. An alternate closed analytic representation of $\mathrm{R}$, originally proposed in [8] and shown to be applicable in in-silico cardiac data in our previous work [9], can speed up the computational efficiency. In particular, the computation of $\mathrm{R}$ is directly proportional to the number of regions $\Omega$, that sum up to represent the whole transformation field.

$$
\mathrm{R}=\frac{1}{\mathrm{P}} \sum_{\Omega} \mathrm{R}_{\Omega}
$$

where $\mathrm{R}$ is sum of individual contributions of the bending energy for each region $\mathrm{R}_{\Omega}$, which can be represented as:

$\mathrm{R}_{\Omega}=\sum_{\left(\delta_{\mathrm{x}}, \delta_{\mathrm{y}}\right)}\left(\mu_{\mathrm{i}}^{\mathrm{T}} \mathrm{V}^{\left(\delta_{\mathrm{x}}, \delta_{\mathrm{y}}\right)} \mu_{\mathrm{i}}+\mu_{\mathrm{j}}^{\mathrm{T}} \mathrm{V}^{\left(\delta_{\mathrm{x}}, \delta_{\mathrm{y}}\right)} \mu_{\mathrm{j}}\right)$ for $\quad\left(\delta_{x}+\delta_{y}=2\right)$ where $\mu_{\mathrm{i}}$ and $\mu_{\mathrm{j}}$ are the B-spline coefficients associated to the relevant control points for $\Omega$ in x and y directions respectively. Importantly, $\mathrm{V}^{\left(\delta_{\mathrm{x}}, \delta_{\mathrm{y}}\right)}$ represent the matrices containing the contribution of the derivative of the BE penalty at every control point $\mu_{\mathrm{ij}}$. For one particular pyramid level, a unique matrix based on the inter-grid spacings $\sigma_{\xi}$ in physical space, constant for that grid resolution can be constructed. This matrix contains the contribution of $\mathrm{BE}$ and it can be precomputed and stored for each grid resolution. In case of 2D images, for every grid resolution three of these matrices were pre-computed and stored. For further details, the reader is referred to [9].

On the end-diastolic (ED) frame (i.e., first frame) of each image sequence, a region of interest (ROI) indicating the myocardial wall was manually contoured. The ROI was sampled into 180 points and six segments were defined according to the recommendation guidelines [10]. The ground truth deformation estimates obtained from the E/M model, were available at the sampled points along the whole cardiac cycle. Subsequently, the ground truth mid-myocardial longitudinal strain $(\epsilon(t))$ was computed, where the strain is defined as deformation of the myocardial length $(\mathrm{L}(\mathrm{t}))$ along the longitudinal direction at any time $t$ in the cardiac cycle and $L_{0}$ being the initial length in the first frame.

$$
\epsilon(t)=\frac{L(t)-L_{0}}{L_{0}}
$$

Ground truth strain curves were obtained at i) each sample of the ROI and, ii) along each of the six segments. For each case the initial length, $L_{0}$ was defined as i) the distance between two consecutive samples of the ROI and ii) the total length of ROI along each segment. The respective end-systole (ES) strains were extracted from both sets of strain curves.

NRIR-based deformation was estimated at all the samples along the ROI. Similar to the ground truth strain curves as described above, two sets of NRIR-based strain curves corresponding to each sample of the ROI and along each segment were obtained. Drift correction was applied to the strain curves, followed by the extraction of the ES strains.

\section{Evaluation}

Tracking performance: To quantify tracking performance, the ideal ES segmental strains as obtained from the E/M model, considered as the reference values were correlated to the NRIR-based ES segmental strains as expressed by the Pearson's correlation coefficient. Bland-Altman (BA) analysis was used to assess the limits of agreement between the ideal and the NRIR-based ES segmental strains. The mean error of the NRIR-based strain (i.e. the bias of the BA anaysis) was tested to be significantly different from zero using a paired t-test and a p-value less than 0.01 was considered to be statistically significant.

Tracking resolution: In an ideal scenario it can be assumed that due to reduced contractility the dysfunctional regions show lower strain values in comparison to the normal regions. The ground truth and the NRIR-estimated dysfunctional areas 

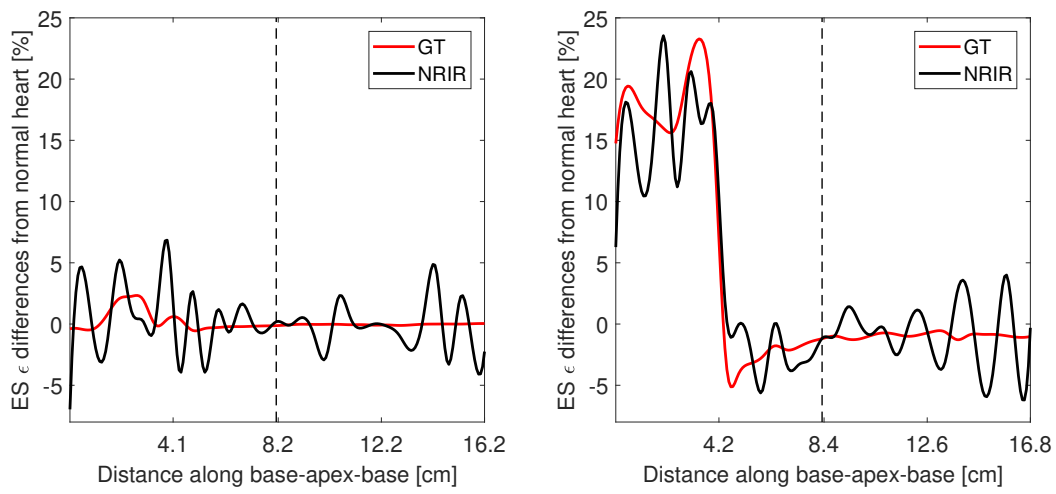

Figure 2. The pixel-wise strain differences between the normal heart model and the models with the smallest (left) and the largest (right) dysfunctional regions for both the ground truth (GT) and the NRIR. The regions with positive strain differences correspond to the dysfunctional regions.

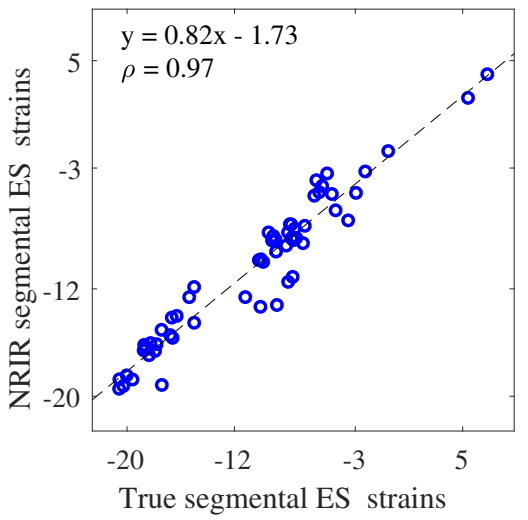

Figure 3. The correlation plot of the NRIR-based versus the true ES segmental strains.

were therefore defined as the samples at the ROI with reduced ES strains with respect to the samples in the respective normal heart model at the same location. Subsequently, for both the ground truth and the NRIR, the size of the dysfunctional areas were determined as the largest spatially continuous dysfunctional regions across the ventricle.

The tracking resolution was evaluated by computing the correlation coefficient between sizes of the dysfunctional area as determined by the NRIR and the ground truth. In addition, to assess dysfunction, sensitivity, specificity and accuracy was computed on a pixel-by-pixel basis and the dysfunctional size was defined to be resolved when accuracy was $>60 \%$. Finally, the receiver operating characteristics (ROC) was computed followed by the estimation of the area under the curve (AUC).

\section{RESULTS}

Figure 1 shows the visual representation of example 3D meshes (models) with the smallest (a) and the largest (b) dysfunctional regions shown in red. The $\mathrm{A} 3 \mathrm{C}$ imaging plane (green) gives a visual estimation of the size of the dysfunctional regions on the imaging plane.

The pixel-wise ES strain differences sampled at the ROI in the normal heart and the heart with the smallest (left) and the

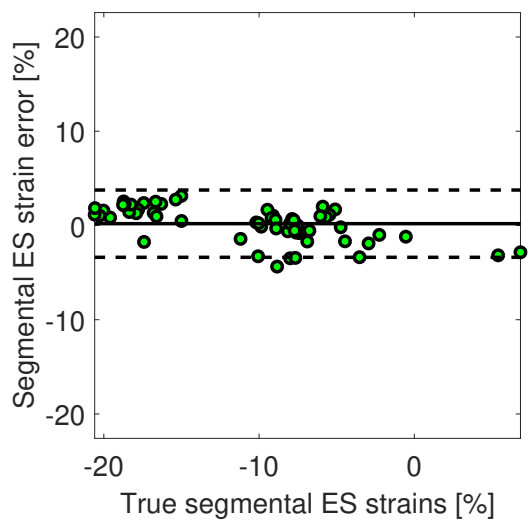

Figure 4. Bland Altman plot for the ES segmental strains.

largest (right) dysfunctional regions is shown in Figure 2. The $\mathrm{x}$-axis denotes the length of the myocardium along the baseapex-base where the dashed vertical line denotes the position of the apex. The ground truth and the NRIR strain differences are shown in red and black respectively, where the regions with positive ES strain differences correspond to the dysfunctional regions.

Figure 3 shows the correlation between the NRIR-based ES segmental strains against the ideal ES segmental strains and a correlation coefficient of 0.97 was obtained. The mean error and the limits of agreement of the NRIR-ES segmental strains with respect to the ground truth measurements were $0.19 \%$ and $-3.37 \cdots 3.76 \%$ respectively (see Figure 4 ), with the difference being statistically significant $(\mathrm{p}<0.01)$.

The correlation coefficient of the NRIR estimated sizes of the dysfunctional area against the ideal sizes was 0.92 (see Figure 5). The pixel-wise accuracy from $1.6 \mathrm{~cm}$ to 5.2 $\mathrm{cm}$ of dysfunctional region increased from $52 \%$ to $82 \%$; while the corresponding AUC increased from 0.49 to 0.95 . A comprehensive overview of our findings for all sizes of dysfunctional areas is presented in Table I. 
Table I

COMPREHENSIVE OVERVIEW OF THE ANALYSIS FOR ALL THE SIZES OF THE DYSFUNCTIONAL AREAS

\begin{tabular}{|c|c||c|c|c|}
\hline \hline View & Size(cm) & Accuracy(\%) & Sensitivity(\%) & Specificity(\%) \\
\hline & 1.6 & 51.9 & 51.7 & 51.9 \\
\cline { 2 - 5 } A3C & 3.4 & 71.9 & 90.84 & 67.2 \\
\cline { 2 - 5 } & 4.3 & 74.2 & 100 & 66.67 \\
\cline { 2 - 5 } & 5 & 81.9 & 100 & 75.4 \\
\hline \hline & 1.9 & 62.23 & 71.43 & 61.38 \\
\cline { 2 - 5 } A4C & 3.5 & 68.1 & 84.21 & 64.53 \\
\cline { 2 - 5 } & 4.6 & 72.86 & 82.69 & 69.62 \\
\cline { 2 - 5 } & 5.2 & 74.29 & 94.23 & 67.7 \\
\hline
\end{tabular}

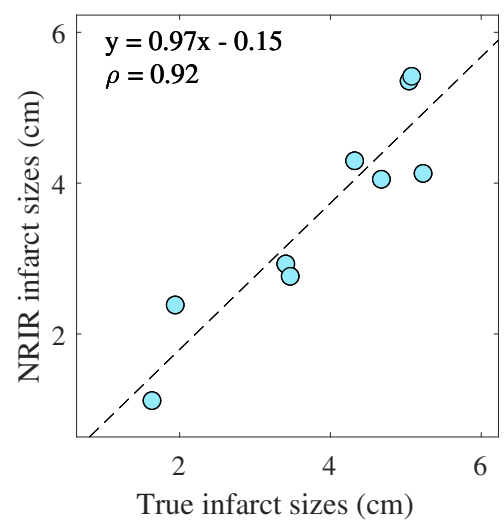

Figure 5. The correlation plot of the sizes of the dysfunctional regions estimated by NRIR against the true ones.

\section{DISCUSSION AND CONCLUSION}

The current study investigated the spatial strain resolution of 2D NRIR on ultra-realistic synthetic B-mode images simulated with eight dysfunctional regions. A reliable NRIR-based tracking performance was supported by a high correlation coefficient of 0.97 (see Figure 3). In addition, a high correlation coefficient of 0.92 (see Figure 5), is suggestive of good tracking resolution.

A difference in the tracking performance was noted between the $\mathrm{A} 3 \mathrm{C}$ images and the $\mathrm{A} 4 \mathrm{C}$ images. A closer look in Table I, shows that for a comparable size of dysfunctional area the accuracy for $\mathrm{A} 3 \mathrm{C}$ was better than that of the $\mathrm{A} 4 \mathrm{C}$. One of the potential explanations could be the out-of-plane motion in the $\mathrm{A} 4 \mathrm{C}$ images which could have been more pronounced due to the generation of the $2 \mathrm{D}$ images from the $3 \mathrm{D}$ mesh. This hypothesis however remains to be proven.

Although we have used ultra-realistic synthetic images, in the future it will be interesting to extend these experiments in clinical in-vivo images, where the corresponding cardiovascular magnetic resonance cardiac data can be used as a reference.

As a conclusion, we have found that the discriminative power of our NRIR algorithm was adequate to resolve dysfunctional areas down to $1.9 \mathrm{~cm}$.

\section{ACKNOWLEDGEMENTS}

This work was supported by funding from the European Research Council, under the European Seventh Framework Pro- gramme (HeartMAPAS; FP7/2007-2013/ERC Grant Agreement number 281748) awarded to the KU Leuven.

\section{REFERENCES}

[1] B. Heyde, R. Jasaityte, D. Barbosa, V. Robesyn, S. Bouchez, P. Wouters, F. Maes, P. Claus, and J. D'hooge, "Elastic image registration versus speckle tracking for 2-D myocardial motion estimation: A direct comparison in vivo," IEEE Trans. Med. Imaging, vol. 32, no. 2, pp. 449-459, 2013.

[2] M. Alessandrini, B. Chakraborty, B. Heyde, O. Bernard, M. De Craene, M. Sermesant, and J. D'hooge, "Realistic vendor-specific synthetic ultrasound data for quality assurance of 2-d speckle tracking echocardiography: Simulation pipeline and open access database," IEEE transactions on ultrasonics, ferroelectrics, and frequency control, vol. 65, no. 3, pp. 411-422, 2018.

[3] S. Marchesseau, H. Delingette, M. Sermesant, M. Sorine, K. Rhode, S. G. Duckett, C. A. Rinaldi, R. Razavi, and N. Ayache, "Preliminary specificity study of the bestel-clément-sorine electromechanical model of the heart using parameter calibration from medical images," Journal of the mechanical behavior of biomedical materials, vol. 20, pp. 259271, 2013.

[4] N. Duchateau, M. De Craene, P. Allain, E. Saloux, and M. Sermesant, "Infarct localization from myocardial deformation: prediction and uncertainty quantification by regression from a low-dimensional space," IEEE transactions on medical imaging, vol. 35, no. 10, pp. 2340-2352, 2016.

[5] D. Rueckert, L. I. Sonoda, C. Hayes, D. L. Hill, M. O. Leach, and D. J. Hawkes, "Nonrigid registration using free-form deformations: application to breast MR images," IEEE Trans. Med. Imaging, vol. 18, no. 8, pp. 712-721, 1999.

[6] H. Lester and S. R. Arridge, "A survey of hierarchical non-linear medical image registration," Pattern recognition, vol. 32, no. 1, pp. 129-149, 1999.

[7] R. H. Byrd, P. Lu, J. Nocedal, and C. Zhu, "A limited memory algorithm for bound constrained optimization," SIAM Journal on Scientific Computing, vol. 16, no. 5, pp. 1190-1208, 1995.

[8] J. A. Shackleford, Q. Yang, A. M. Lourenço, N. Shusharina, N. Kandasamy, and G. C. Sharp, "Analytic regularization of uniform cubic B-spline deformation fields," in MICCAI 2012. Springer, 2012, vol. 7511, pp. 122-129.

[9] B. Chakraborty, B. Heyde, M. Alessandrini, and J. D'hooge, "Fast myocardial strain estimation from 3D ultrasound through elastic image registration with analytic regularization," in SPIE Medical Imaging. International Society for Optics and Photonics, 2016, pp. $979006-$ 979006.

[10] J.-U. Voigt, G. Pedrizzetti, P. Lysyansky, T. H. Marwick, H. Houle, R. Baumann, S. Pedri, Y. Ito, Y. Abe, S. Metz et al., "Definitions for a common standard for 2D speckle tracking echocardiography: consensus document of the EACVI/ASE/Industry task force to standardize deformation imaging," European Heart Journal-Cardiovascular Imaging, vol. 16 , no. 1 , pp. $1-11,2014$. 